\title{
Asociación de adenocarcinoma de esófago y cáncer de mama en un varón con enfermedad de Madelung
}

\author{
P. LINARES TORRES, C. CASTAÑÓN LÓPEZ1 ${ }^{1}$ C. LLANO ALONSO ${ }^{2}$, M. ÁLVAREZ \\ POSADILLA, S. VIVAS ALEGRE, J. ESPINEL DÍEZ, M. T. RIBAS ARINO ${ }^{3}$
}

Sección de Aparato Digestivo, Unidad de Oncología Médical, Servicio de Medicina Interna ${ }^{2}$ de Anatomía Patológica ${ }^{3}$ del Hospital de León.

ASSOCIATION OF ADENOCARCINOMA OF ESOPHAGUS AND BREAST CANCER IN A MALE WITH MADELUNG' DISEASE

\begin{abstract}
RESUMEN
La aparición de un cáncer de esófago en mujeres que previamente han recibido radioterapia como tratamiento de un cáncer de mama es un hecho conocido aunque poco frecuente. El riesgo de aparición de la segunda neoplasia es mayor cuando han transcurrido diez o más años desde la exposición a la radiación. La estirpe histológica de la neoplasia de esófago más frecuente es la de carcinoma de células escamosas. Se presenta un caso de adenocarcinoma de esófago en un varón, diagnosticado de lipomatosis bilateral simétrica o enfermedad de Madelung, que recibió radioterapia tres años antes por presentar un cáncer de mama.
\end{abstract}

PALABRAS CLAVE: Cáncer de esófago. Cáncer de mama. Enfermedad de Madelung.

\begin{abstract}
The development of a cancer of the esophagus in women who previously had received radiotherapy for breast cancer is a known although infrequent event. The risk of apparition of the second neoplasia is greater in women who survive at least ten years after the exposition to the radiation. Squamous cell carcinoma is the histological subtype more frequent. We report a case of adenocarcinoma of the esophagus in a man diagnosed of benign symmetrical lipomatosis (Madelung' disease), who had received adjuvant radiotherapy three years before for breast cancer.
\end{abstract}

KEY WORDS: Esophagus cancer. Breast cancer. Madelung' disease

Linares Torres P, Castañón López, C, Llano Alonso C, Álvarez Posadilla M, Vivas Alegre S, Espinel Díez J, Ribas Arino MT. Asociación de adenocarcinoma de esófago y cáncer de mama en un varón con enfermedad de Madelung. An Med Interna (Madrid) 2006; $133-135$.

\section{INTRODUCCIÓN}

Diversos trabajos han sugerido que existe una relación entre la administración de radioterapia para el tratamiento de determinados tumores del tórax y cuello y la aparición ulterior de un cáncer de esófago (1-3). La exposición a la radiación puede ser una causa de cancerización de campo en el tracto digestivo superior.

En un reciente estudio sobre incidencia de cáncer de esófago como segundo tumor primario en mujeres tratadas previamente por cáncer de mama (4), los autores demostraron que el subgrupo de pacientes que habían recibido radioterapia tenía un riesgo aumentado de desarrollar durante el seguimiento un carcinoma de esófago. Este riesgo se incrementaba para la variante de carcinoma esofágico de células escamosas y cuando el tiempo de evolución era de 10 años o más desde la administración de la radioterapia.
Presentamos el caso de un varón con una lipomatosis simétrica benigna o enfermedad de Madelung, que desarrolló un cáncer de mama por el que recibió radioterapia y que fue diagnosticado tres años después de un adenocarcinoma de esófago.

\section{CASO APORTADO}

Varón de 68 años de edad que presenta disfagia de 6 meses de evolución, de carácter progresivo, inicialmente frente a alimentos sólidos y posteriormente a líquidos, con pérdida de unos $10 \mathrm{~kg}$ de peso. El paciente es un agricultor jubilado, bebedor de unos $100 \mathrm{~g}$ alcohol/día y fumador 20 cigarrillo/día, hasta hace 3 años. Había sido diagnosticado de una enfermedad de Madelung hacía 10 años y de un adenocarcinoma ductal infiltrante de mama derecha (pT4N1Mx) hacía 3 años, realizándose mastectomía radical modificada y linfadenectomía axilar con afectación de 10 de 17 ganglios aislados. Reci-

Trabajo aceptado: 26 de octubre de 2005 
bió tratamiento con poliquimioterapia y radioterapia tras la cirugía y posteriormente se indicó tamoxifeno oral de mantenimiento. No tenía otros antecedentes personales de interés ni tampoco antecedentes familiares de enfermedades oncológicas. En el examen físico llamaba la atención la presencia de lipomas simétricos en la región cervical con aspecto de cuello de búfalo y una cicatriz de mastectomía derecha, sin otras alteraciones. En el hemograma, destacaba una anemia leve normocítica normocrómica y la bioquímica completa que incluía las enzimas hepáticas no mostraba alteraciones significativas. Se practicó un esofagograma (Fig. 1) que puso de manifiesto una estenosis de contorno irregular y espiculado del esófago distal, con desestructuración de la mucosa y dilatación preestenótica, compatible con neoplasia de esófago. La gastroscopia confirmó la presencia de una estenosis ulcerada de esófago distal que impedía el franqueo del endoscopio hacia estómago y permitió la toma de biopsias que fueron compatibles con adenocarcinoma de esófago moderadapobremente diferenciado sobre un esófago de Barrett (Fig. 2). En el TAC cervico-torácico se observaban varios lipomas a ambos lados del cuello, tanto en el triángulo anterior como posterior, por delante del músculo esternocleidomastoideo. No había adenopatías laterocervicales y sí un esófago dilatado con una masa concéntrica en esófago distal que producía un estrechamiento de la luz. En la ecografía abdominal se detectaron múltiples lesiones focales hipoecogénicas, de diferentes tamaños, localizadas en ambos lóbulos, sugestivas de

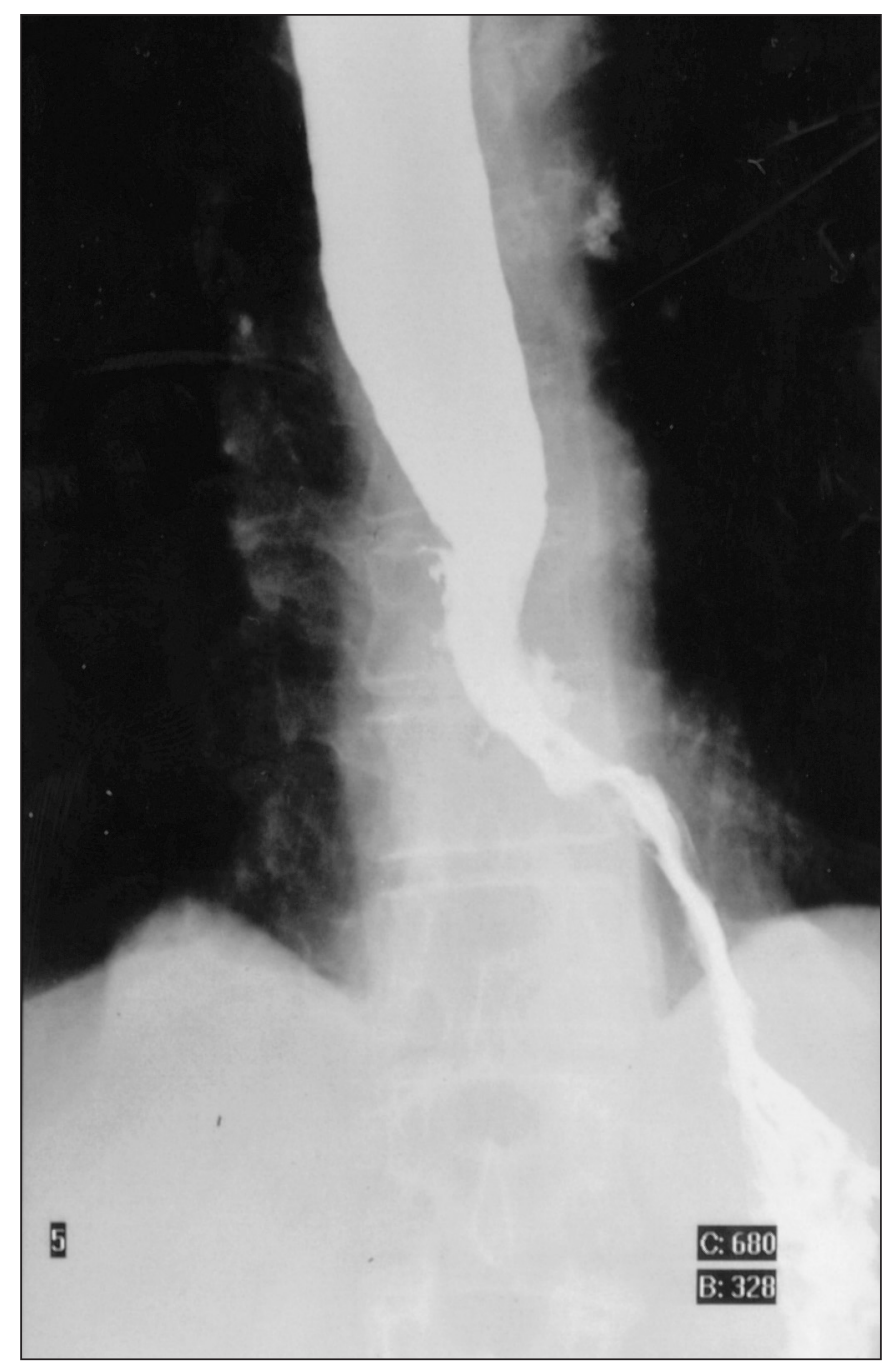

Fig. 1. Estenosis de contorno irregular y espiculado del esófago distal, con desestructuración de la mucosa y dilatación preestenótica, compatible con neoplasia de esófago.

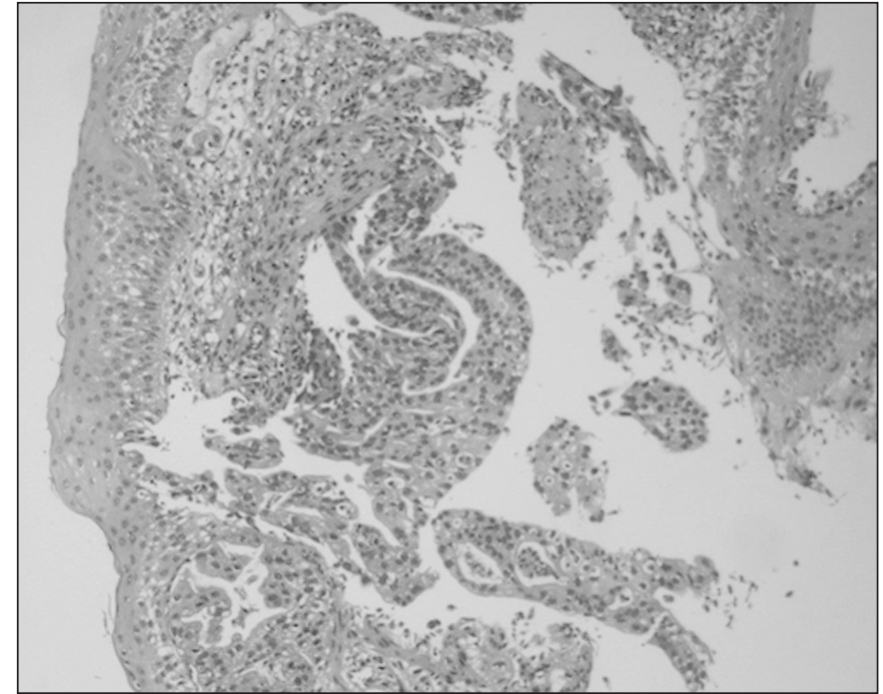

Fig. 2. Corte histológico que muestra una mucosa de esófago infiltrada por un tumor epitelial altamente sugestivo de adenocarcinoma moderadamente-poco diferenciado sobre esófago de Barreto (H-Ex 20)

metástasis hepáticas, sin presencia de líquido libre. Al paciente se le colocó una endoprótesis esofágica y estaba pendiente de realización de PAAF hepática para intentar aclarar el origen mamario o esofágico de las lesiones focales hepáticas y así decidir el tipo de tratamiento quimioterápico. Sin embargo, presentó varios episodios de hematemesis y deterioro de su estado general, que contraindicaron la administración de quimioterapia, realizándose un tratamiento sintomático paliativo. El paciente finalmente falleció por una hematemesis masiva, un mes después del diagnóstico de la neoplasia esofágica.

\section{DISCUSIÓN}

Este caso es peculiar por diferentes aspectos. En primer lugar, el paciente tiene una lipomatosis bilateral simétrica o enfermedad de Madelung, caracterizada por el acúmulo subcutáneo difuso, progresivo y simétrico de grasa alrededor del cuello, espalda y región interescapular ocasionando un fenotipo característico (5). A pesar de no ser una enfermedad excesivamente rara, tiene una incidencia aproximada de 4/100.000 habitantes, con una relación hombre/mujer de 15-30:1. Se la ha relacionado con el consumo excesivo de alcohol en más del $90 \%$ de los casos, aunque también se reconocen otras posibles asociaciones a metabolopatías e incluso formas hereditarias (6,7). El carácter progresivo y simétrico de este cuadro clínico en un varón con etilismo crónico, el fenotipo característico y la ausencia de malignidad permiten el diagnóstico de esta entidad.

En nuestro conocimiento, no hay ningún caso previo descrito de enfermedad de Madelung y cáncer de mama. El cáncer de mama que afecta al hombre es una enfermedad infrecuente, con una incidencia de 1 caso/100.000 hombres, representando el $0,6 \%$ de todos los cánceres de mama y menos del $1 \%$ de todos los tumores malignos del hombre, si bien parece que su incidencia está aumentando en los últimos años (8). La presentación clínica del cáncer de mama en el hombre suele ser diferente al de la mujer, con una edad media al diagnóstico mayor, 67 años para el hombre y 62 para la mujer; y un estado de la enfermedad más avanzado al diagnóstico, con mayor tamaño tumoral y presencia de afectación ganglionar (9), como sucedía en este caso. 
Dentro de los factores de riesgo para cáncer de mama en hombres se encuentran las alteraciones en el balance entre estrógenos y andrógenos, además de otras condiciones como testículo no descendido, síndrome de Klinefelter, orquiectomía, etc. La obesidad, con un aumento en la producción periférica de estrógenos y la cirrosis hepática que implica un estado hiperestrogénico, serían otros posibles factores (9).

Recientemente se sabe que el consumo de alcohol aumenta el riesgo de cáncer de mama un $7 \%$ por cada $10 \mathrm{~g}$ de alcoho/d consumido en la mujer y un $16 \%$ en el hombre (10). Posiblemente, la enfermedad de Madelung, al tratarse en realidad de una lipodistrofia y al igual que se relaciona con un incremento en la resistencia de la insulina y otras alteraciones metabólicas, podría ocasionar un disbalance estrogénico, que junto con el consumo excesivo de alcohol podría condicionar un mayor riesgo para la aparición de cáncer de mama en este paciente.

El desarrollo de un cáncer de esófago como segundo tumor primario es un hecho conocido, aunque infrecuente y que se ha relacionado con el consumo de alcohol, tabaco, radioterapia, etc. (11). La descripción de algunos casos aislados (1-3) y sobre todo, los resultados de un estudio relativamente reciente, han sugerido que existe un riesgo aumentado para el desarrollo de un cáncer de esófago en mujeres que recibieron previamente radioterapia como parte del tratamiento de un cáncer de mama (4). El efecto carcinogénico de la radioterapia en estos casos se sustenta por dos hechos: a) el riesgo de cáncer de esófago está aumentado en las pacientes con cáncer de mama que recibieron radioterapia y quimioterapia, pero no en aquellas otras mujeres que no sólo recibieron quimioterapia; y b) este riesgo es mayor cuando se radiaba la parte interna de la mama.

El patrón de aparición de un carcinoma escamoso de esófago después de la irradiación de un cáncer de mama sigue el modelo de carcinogénesis inducida por la radioterapia, con un riesgo relativo de 1,37 cuando el periodo de seguimiento es inferior a 5 años y de 5,42 cuando este periodo es superior a 10 años. Para el adenocarcinoma de esófago, el riesgo relativo es de 4,22 después de 10 años de seguimiento (4).

Aunque la estirpe histológica más común del cáncer de esófago cuando se trata de un segundo tumor primario es la escamosa, tampoco es infrecuente la variante adenocarcinoma (4) y quizás ésta, pueda aparecer de forma más precoz en el seguimiento. En nuestro caso, el adenocarcinoma de esófago asentaba sobre un esófago de Barrett, que incrementa el riesgo de desarrollo de un adenocarcinoma de esófago entre un 0,5 y $1 \%$ anual $(12,13)$.

Otros factores de riesgo reconocidos para el desarrollo de una neoplasia esofágica tanto de células escamosas como adenocarcinoma, que presentaba nuestro paciente, son el consumo excesivo de alcohol y tabaco. La enfermedad de Madelung no parece que esté relacionado con un mayor riesgo para el desarrollo de un cáncer de esófago. Sin embargo, la presencia de un esófago de Barrett como manifestación de una enfermedad por reflujo gastroesofágico crónico y oligosintomático y la irradiación torácica, podrían ser otros factores de riesgo que habrían interactuado con los anteriores e incluso potenciado, favoreciendo la aparición más precoz del cáncer de esófago, con un intervalo entre ambos procesos neoplásicos de tan sólo 3 años.

\section{Bibliografía}

1. Goffman TE, McKeen EA, Curtis RE, Schein PS. Esophageal carcinoma following irradiation for breast cancer. Cancer 1983; 52: 1808-9.

2. Shousha S, Fawcett A, Luqmani YA, Theodorou N. Multifocal squamous cell carcinoma of the oesophagus following radiotherapy for bilateral breast carcinoma. J Clin Pathol 2001; 54: 718-20.

3. Scholl B, Reis ED, Zouhair A, Chereshnev I, Givel JC, Gillet M. Esophageal cancer as second primary tumor after breast cancer radiotherapy. Am J Surg 2001; 182: 476-80.

4. Ahsan H, Neugut AI. Radiation therapy for breast cancer and increased risk for esophageal carcinoma. Ann Intern Med 1998; 128: 114-7.

5. Matamala Gimeno C, Perez Calvo JI, Amores Ferreras M. Multiple symmetric lipomatosis. An Med Interna (Madrid) 1994; 11: 95-9.

6. Haap M, Siewecke C, Thamer C, Machann J, Schick F, Haring HU, Szeimies RM, Stumvoll M. Multiple symmetric lipomatosis: a paradigm of metabolically innocent obesity? Diabetes Care 2004;27: 794-5.

7. Payne CE. Hereditary Madelung's disease. J R Soc Med 2000;93: 194-5.
8. Giordano SH, Cohen DS, Buzdar AU, Perkins G, Hortobagyi GN. Breast carcinoma in men: a population-based study. Cancer 2004; 101: 51-7.

9. Giordano SH, Buzdar AU, Hortobagyi GN. Breast cancer in men. Ann Intern Med 2002; 137: 678-87.

10. Guenel P, Cyr D, Sabroe S, Lynge E, Merletti F, Ahrens W, et al. Alcohol drinking may increase risk of breast cancer in men: a European population-based case-control study. Cancer Causes Control 2004; 15: 571-80.

11. van Halteren HK, Taal BG, van Tinteren H, van Leeuwen FE. Risk factors for the development of oesophageal cancer as a second primary tumour. Eur J Cancer 1995; 31A: 1836-9.

12. Spechler SJ, Goyal RK. Barrett's esophagus. N Engl J Med 1986; 315 : 362-71.

13. Chang JT, Katzka DA. Gastroesophageal reflux disease, barrett esophagus, and esophageal adenocarcinoma. Arch Intern Med 2004; 164 : 1482-8. 